\title{
Enhancing resistance of silk fibroin material to enzymatic degradation by cross-linking both crystalline and amorphous domains
}

Filippo Valente ${ }^{a *}$, Benjamin J. Allardyce ${ }^{\mathrm{b} *}$, Matt S. Hepburn ${ }^{\mathrm{c}, \mathrm{d}}$, Philip Wijesinghe ${ }^{\mathrm{c}, \mathrm{de}}$, Sharon L. Redmond ${ }^{\mathrm{a}}$, Jingyu Chen ${ }^{\mathrm{b}}$, Brendan F. Kennedy ${ }^{\mathrm{c}, \mathrm{d}}$, Rangam Rajkhowa ${ }^{\mathrm{b}}$, Marcus D. Atlas ${ }^{\mathrm{a}}$, Xungai Wang ${ }^{\mathrm{b}}$ and Rodney J. Dilley, f,,**

${ }^{a}$ Ear Science Institute Australia; Subiaco, Australia and Ear Sciences Centre, Faculty of Health and Medical Sciences, The University of Western Australia, Nedlands, Australia.

${ }^{b}$ Deakin University, Institute for Frontier Materials, Geelong, Australia.

cBRITElab, Harry Perkins Institute of Medical Research, QEII Medical Centre, Nedlands and Centre for Medical Research, The University of Western Australia, Perth, Australia.

${ }^{d}$ Department of Electrical, Electronic and Computer Engineering, School of Engineering, The University of Western Australia, Perth, Australia

eSUPA, School of Physics and Astronomy, University of St Andrews, UK

${ }^{f}$ Centre for Cell Therapy and Regenerative Medicine, School of Biomedical Sciences, The University of Western Australia, Nedlands Australia.

*First co-authors

** Corresponding author: rodney.dilley@earscience.org.au. Ear Science Institute Australia, 8 Verdun Street, NEDLANDS WA 6009. 


\begin{abstract}
Silk fibroin (SF) membranes are finding widespread use as biomaterial scaffolds for a range of tissue engineering applications. The control over SF scaffold degradation kinetics is usually driven by the proportion of SF crystalline domains in the formulation, but high $\beta$-sheet membranes are brittle and still contain amorphous domains, which are highly susceptible to enzymatic degradation. In this work, photo-cross-linking of SF using a ruthenium-based method, and with the addition of glycerol, was used to generate robust and flexible SF membranes for long-term tissue engineering applications requiring slow degradation of the scaffolds. The resulting mechanical properties, protein secondary structure and degradation rate were investigated. In addition, the cytocompatibility and versatility of porous micropatterning of SF films was assessed. The photo-cross-linking reduced enzymatic degradation of SF in vitro without interfering with the $\beta$-sheet content of the SF material, while adding glycerol to the composition grants flexibility to the membranes. By combining these methods, the membrane resistance to protease degradation was significantly enhanced compared to either method alone, and the SF mechanical properties were not impaired. We hypothesise that photo-cross-linking protects the SF amorphous regions from enzymatic degradation and complements the natural protection offered by $\beta$-sheets in the crystalline region. Overall, this approach presents broad utility in tissue engineering applications that require a long-term degradation profile and mechanical support.
\end{abstract}

Abbreviations: Pure SF membranes, P-SM; Pure SF membranes with glycerol, P-SM-G; Photo-cross-linked SF membranes, CL-SM; Photo-cross-linked SF membranes with glycerol, CL-SM-G

Keywords: silk fibroin, degradation, cross-link, tissue engineering. 


\section{Introduction}

Biomaterial scaffolds for tissue engineering can be specifically designed to address the required shapes and physico-chemical properties for a specific application. Silk fibroin (SF) is a fibrous protein that has been identified as a widely suitable biomaterial due to its biocompatibility, mechanical strength, tuneable degradation, oxygen and water vapour permeability ${ }^{1-5}$. Numerous tissue engineering applications have been published, including by our group to provide mechanical support and suitable acoustic properties for ear drum replacement ${ }^{6}$ and by others for corneal wound healing films ${ }^{7}$, skin and cartilage regeneration ${ }^{8-9}$ and vascular conduits for anastomosis 10 among others. SF is a self-assembling, hierarchical protein composed of a $\beta$-sheet nanocrystals within a network of semi-amorphous domains ${ }^{11}$. During natural silk spinning, these regions are arranged into nanofibrils, which, in turn pack together to form the silk filament. Hydrogen bonding, primarily within anti-parallel $\beta$-sheets, form physical cross-links that gives fibroin its mechanical strength while the amorphous regions provide elasticity. The highly level of structural organisation, including nanocrystal size and their arrangement within the nanofibrils gives silk the strain hardening behaviour responsible for its exceptional toughness ${ }^{12}$. However, when SF is regenerated from the dissolved form of protein into solid format, there is a limited control over the level of self-assembly of the protein chains and, resulting material, once dried, tends to be very brittle ${ }^{13-15}$.

Regenerated SF material is not water stable and partially dissolves in water due to poor content of $\beta$-sheet crystals. Hence a variety of post-processing methods are used to enhance $\beta$-sheet content of SF materials, including treatment with alcohol, ${ }^{13,16}$ water vapour ${ }^{17}$ or stretching ${ }^{18}$. Such treatments reduce SF solubility in water and coincidentally increases resistance to enzymatic degradation. These methods do not form any chemical cross links but induce high density hydrogen bonding, which contributes to $\beta$-sheet formation ${ }^{19}$. On the other hand, enzymatic methods have also been reported for SF modification under mild conditions, including the use of laccase ${ }^{20}$, tyrosinase ${ }^{21}$, and HRP ${ }^{22-23}$, which all target the covalent crosslink of tyrosine residues, endowing functionalization of SF, if coupled with antibacterial or antioxidant agents, and can modify the chemistry of protein matrices without breaking the peptide bonds.

Our previous work focused on making membranes using a combination of SF in formic acid and glycerol that were flexible and degradation-resistant for applications that required slower 
degradation profiles ${ }^{24}$. Glycerol was used as a plasticizing agent ${ }^{25}$ that at concentration $\geq 20 \%$ $\mathrm{w} / \mathrm{w}$ induces a conformation change of the SF proteins, making the resulting membrane water insoluble ${ }^{26-27}$. However, when using natural physical cross-linking for SF that is based on hydrogen bonding, only a limited sequence in SF protein can form such bonds and compact pleated $\beta$-sheet structure. Many amino acid residues which have bulky side chains cannot form hydrogen bonded tight structure, such that nearly half of the SF secondary structure is dominated by amorphous random coil or $\alpha$-helical conformations. Enzyme attack is very rapid in these regions, particularly in regenerated SF, leading to their fast degradation due to restricted amount of $\beta$-sheet that can be formed ${ }^{28-29}$. There is, therefore, less control over the structure of random coil amorphous parts of the SF.

In an effort to improve the control over the SF degradation properties, we used photo-crosslinking of SF solutions based on tris(2,20-bipyridyl)dichlororuthenium(II) (Ru(II)(bpy) ${ }^{2+}$. This cross-linking strategy, which was first described 20 years ago ${ }^{30}$, has been used in a range of proteins including resilin ${ }^{31}$, fibrinogen ${ }^{32}$, gelatin ${ }^{33}$, collagen ${ }^{33}$ and, more recently, silk ${ }^{5}$. In the presence of white light, $\left(\mathrm{Ru}(\mathrm{II})(\mathrm{bpy}){ }_{3}{ }^{2+}\right.$ is photolyzed in the presence of a persulfate (commonly sodium persulfate, SPS, or ammonium persulfate, APS) generating Ru(III) and a sulfate radical. This highly reactive $\mathrm{Ru}(\mathrm{III})$ intermediate then oxidises reactive side chains in proteins such as tyrosine. This produces a tyrosyl radical which, in turn, can react with other residues such as other tyrosines (via arene coupling to form dityrosine); another possible reaction is between a tyrosine residue and another side chain such as cysteine or lysine (via a heteroatom-arene linkage) ${ }^{30}$. Within silk specifically, dityrosine cross-linking is more likely since tyrosine $(5.3 \mathrm{~mol} \%)$ is much more abundant than cysteine $(0.1 \mathrm{~mol} \%)$ or lysine $(0.2 \%)$ 34-35. Unlike physical cross-linking, which occur primarily within the crystalline $\beta$-sheet forming regions, chemical cross-linking is possible in both crystalline and amorphous regions, wherever tyrosine is present. It is therefore possible that the addition of chemical crosslinks can affect silk's degradation profile by providing protection to the amorphous regions of silk that are susceptible to enzymatic degradation.

The choice of a photoinitiator is important because its specific absorption peak dictates the wavelength of light that must be used ${ }^{36}$. Photo-cross-linking using the ruthenium method has the advantage of being quickly activated using low energy visible light, and the crosslinking density can be controlled by the amount of initiator present in the solution ${ }^{37}$. The use of visible light is preferable to the other widely used UV-activated cross-linking methods since it offers greater cross-linking efficiency and deeper penetration through the material ${ }^{36,38}$ and it is not 
toxic to cells even when cross-linking hydrogels containing live cells ${ }^{39}$. This significantly increases the usefulness of the system since it makes it suitable for other fabrication methods, such as live cell bioprinting ${ }^{40}$. Photo-cross-linking using ruthenium has been tested for a range of applications, from stabilizing collagen and gelatin to being used as a tissue sealant ${ }^{33,41-42}$ and more recently to prepare hydrogels from Bombyx mori $\mathrm{SF}^{5,28}$.

In this work, we propose an approach to control the degradation rate of the SF biomaterials based on the combination of formic acid and glycerol with the addition of ruthenium photocross-linking, that differs significantly from the established methods of controlling degradation rate by modifying just the $\beta$-sheet content of the material $24,27,43-44$. The result is the production of membranes with exceptional resistance to enzymatic degradation, but that retain similar mechanical properties to un-cross-linked membranes. 


\section{Materials and methods}

\section{Preparation of lyophilised SF foam}

Reeled, undegummed fibers from bivoltine Bombyx mori SFworms (purchased from Karnataka, India) were degummed for $30 \mathrm{~min}$ at $98^{\circ} \mathrm{C}$ using $2 \mathrm{~g} / \mathrm{L}$ sodium carbonate (Sigma-Aldrich, St. Louis, MO, USA), and $1 \mathrm{~g} / \mathrm{L}$ unscented olive oil soap (Vasse Virgin, Wilyabrup, Western Australia, Australia). After degumming, fibers were rinsed with Milli- $Q$ water and dried for 24 $\mathrm{h}$ at $40{ }^{\circ} \mathrm{C}$. The degummed fibroin was then dissolved for $5 \mathrm{~h}$ at $60{ }^{\circ} \mathrm{C}$ using $9.3 \mathrm{M}$ lithium bromide at a material:liquor ratio of $1: 7.5$. The dissolved SF was dialyzed for 3 days at $4{ }^{\circ} \mathrm{C}$ using Slide-A-Lyzer ${ }^{\mathrm{TM}}$ G2 $10 \mathrm{KDa}$ MWCO dialysis cassette (Thermo Scientific Pierce, Rockford, IL, USA). The SF concentration after dialysis was between $4 \%$ and $5 \% \mathrm{w} / \mathrm{v}$ as determined by gravimetric analysis. The SF solution from each batch was diluted to $4 \% \mathrm{w} / \mathrm{v}$, separated into $20 \mathrm{~mL}$ aliquots and frozen overnight at $-80{ }^{\circ} \mathrm{C}$ before being transferred to a prechilled FreeZone freeze-drier (Labconco, Kansas City, MO, USA) and dried for 3 days at $80{ }^{\circ} \mathrm{C}$.

\section{Production of SF membranes}

SF foam was sliced using a scalpel and dissolved for 1 hour at $30{ }^{\circ} \mathrm{C}$ in $99 \%$ formic acid (Thermo Scientific, Waltham, MA, USA) to achieve a final SF concentration in solution of $5 \%$ w/v. For preparations including glycerol, the SF solution was added to a tube containing preweighed glycerol to a final concentration of $40 \% \mathrm{w} / \mathrm{w}$ relative to the weight of SF in solution. The production of photo-cross-linked SF films, was the results of a two-phase process: a gelation phase and an evaporation phase. Stock solutions of $2 \mathrm{mM}$ Tris(2,2'bipyridyl)dichlororuthenium(II) hexahydrate (ruthenium) (Sigma-Aldrich,) and $20 \mathrm{mM}$ sodium persulfate (SPS) (Sigma-Aldrich) were made using Milli- $Q$ water. In order to produce photo-cross-linked SF films these photo-reagents were added to the SF/glycerol solution at final dilution of $40 \mu \mathrm{L} / \mathrm{mL}$ (for the ruthenium) and $10 \mu \mathrm{L} / \mathrm{mL}$ (for the SPS) and $3.5 \mathrm{ml}$ of the mixed solution was cast into sterile $60 \mathrm{~mm}$ diameter Petri dishes. To induce the gelation reaction, the solutions were exposed to a $22 \mathrm{~W}$ visible light source for 10 minutes at approximately $30 \mathrm{~cm}$ distance. To produce un-cross-linked SF films, $3.5 \mathrm{ml}$ of SF/glycerol solution without photo-reagents was cast into sterile $60 \mathrm{~mm}$ diameter Petri dishes. All the Petri 
dishes were left to dry in a fume hood overnight, ensuring enough time to allow the crosslinking reaction to complete.

Table 1: SF membranes composition, abbreviation list and dry thickness

\begin{tabular}{ll}
\hline Membrane composition & Abbreviation \\
\hline Pure SF solution & P-SM \\
\hline Pure SF solution $+40 \%$ w/w glycerol & P-SM-G \\
\hline Photo-cross-linked SF solution & CL-SM \\
\hline Photo-cross-linked SF solution $+40 \% \mathrm{w} / \mathrm{w}$ glycerol & CL-SM-G
\end{tabular}

\section{SF membrane secondary structure}

The proportion of crystalline motifs ( $\beta$-sheet and turn) and amorphous motifs ( $\alpha$ - helix and random coil) was measured in each membrane type using a Vertex 70 Fourier transform infrared (FTIR) spectrophotometer (Bruker, Billerica, MA, USA) by an established method ${ }^{45}$. Scans were taken in absorbance mode over the range of 4000 to $600 \mathrm{~cm}^{-1}$. A total of 64 scans were coadded at a resolution of $4 \mathrm{~cm}^{-1}$ to produce the spectra. A total of 3 membranes of each type were measured, with 4 scans taken per membrane (edge of the membrane, top surface, edge of the membrane bottom surface, centre of the membrane top surface, centre of the membrane bottom surface) over 3 separate films, for a total of 12 measurements per membrane type. The top and bottom surface scans were averaged, and the amide I region (1705 to 1595 $\mathrm{cm}^{-1}$ ) was subjected to deconvolution and curve fitting using 7 known conformational peaks as described previously ${ }^{45-46}$. The relative peak area of each of these 7 deconvoluted peaks was used to determine the $\%$ content of side chain, $\beta$-sheet, random coil, $\alpha$-helix and $\beta$ - turn. The $\%$ peak area values were expressed as the mean \pm standard deviation of 6 measurements (centre 
and edge region of 3 separate membranes). The averaged scan of all samples per membrane type was also plotted after deconvolution.

\section{Tensile mechanical properties}

The tensile properties of the cross-linked membranes were measured using a model 5967 tester (Instron, Norwood, MA, USA) with a $100 \mathrm{~N}$ load cell. Films were cut into $5 \mathrm{~mm}$ wide strips, conditioned for at least $48 \mathrm{hrs}$. at $20^{\circ} \mathrm{C} \pm 2{ }^{\circ} \mathrm{C}$ and $65 \% \pm 2 \%$ relative humidity and then tested until break using a gauge length of $15 \mathrm{~mm}$ and an extension rate of $150 \mathrm{~mm} / \mathrm{min}$. A minimum of 20 strips were tested across at least 3 membranes; tensile properties were expressed as mean \pm standard deviation of these measurements.

\section{Degradation analysis}

Membranes were tested using an accelerated in vitro enzymatic degradation assay with protease XIV from Streptomyces griseus (Sigma-Aldrich) using a modified method based on previous work [20]. Briefly, dry SF membrane samples were weighed and treated with 1 $\mathrm{mg} / \mathrm{mL}$ protease solution (equal to $3.5 \mathrm{U} / \mathrm{mL}$ ) dissolved in $0.1 \mathrm{M}$ phosphate saline buffer (PBS) at $\mathrm{pH} 7.4$, in ratio 1:150 w/w. Controls were incubated in PBS alone. The membranes were incubated at $37^{\circ} \mathrm{C}$ at $50 \mathrm{rpm}$ in a shaker. Samples were collected at different times $(0,1,2,4$, $6,12,24$ hours) and analysed using optical coherence tomography (OCT), which provides volumetric imaging of the sample microstructure based on its optical back-scattering properties. Volumetric OCT scans were acquired using a fiber-based spectral-domain OCT system (Telesto 320, Thorlabs Inc., USA). The light source is a superluminescent diode with a mean wavelength of $1300 \mathrm{~nm}$ and a spectral bandwidth of $170 \mathrm{~nm}$. The measured axial and lateral resolution (in air) is $4.8 \mu \mathrm{m}$ and $7.2 \mu \mathrm{m}$ respectively. Scans were acquired by taking 1000 by 1000 axial line scans across a $10 \mathrm{~mm}$ by $10 \mathrm{~mm}$ lateral field of view. For each sample, the SF thickness was determined from the OCT scan using morphological image processing.

Cell culture, viability assay and morphological analysis

For cell culture and viability experiments, $7 \mathrm{~mm}$ diameter disks of each SF membrane were harvested using a biopsy punch. Each membrane was washed in $70 \%$ ethanol, then rinsed 3 times in PBS and equilibrated in specific growth medium overnight before being used for cell 
culturing, allowing the release of the photo-reagent in the SF membranes (Supplementary Figure 1). Each membrane was placed in a well of a 96 well plate (Corning, New York, USA) and cells were seeded on top in $100 \mu \mathrm{L}$ of medium. For cell culture and viability assays, 10000 cells were seeded onto each membrane and analysed after 24 hours and 7 days of culturing. As a control, an equal amount of cells was seeded into empty wells of the same plate and cultured in the same conditions. Each treatment group had $7 \mathrm{~mm}$ films tested in triplicate and from 3 different cross-linked SF membranes.

Human tympanic membrane keratinocytes (hTMk) derived from normal human tympanic membrane explant harvested as previously described ${ }^{47-48}$. The St John of God HealthCare Ethics Committee approved collection of excess of tympanic membrane tissue undergoing otological surgical procedures at St John of God Hospital (Subiaco, 6008 WA, Australia). The keratinocytes were cultured in Dulbecco's Modified Eagle Medium (DMEM) containing 4.5 $\mathrm{g} / \mathrm{L}$ D-glucose (Gibco, Australia), supplemented with $10 \%$ foetal bovine serum (FBS, Bovogen, Australia), $1 \%$ Penicillin-Streptomycin, and incubated at $37^{\circ} \mathrm{C}$ with $5 \% \mathrm{CO}_{2}$. Medium was replaced every 2-3 days.

Cell-scaffold interaction was assessed by staining the cells with fluorescent markers and observing them with optical fluorescence microscopy and with confocal microscopy. Samples were rinsed in Phosphate-Buffered Saline (PBS) (Gibco), fixed in 4\% paraformaldehyde for 10 minutes and then rinsed again in PBS 3 times for 5 minutes. The scaffolds containing hTMk were then incubated with fluorescent nuclear dye (DAPI; Molecular Probes, USA) at concentration of 1:1000 for 20 minutes, rinsed with PBS, cover slipped and imaged. Images were capture using an optical fluorescence microscope (BX70 Olympus, Nikon, Japan).

\section{Scanning Electron Microscopy}

Scaffolds containing cells were fixed overnight with glutaraldehyde, rinsed in PBS before dehydration with ethyl alcohol (50\%, 70, 95, 99\% and 100\%) for 30 minutes each. Scaffolds were then chemically dried in a mixture of Hexamethyldisilizane (HDMS) (Sigma, Australia) and ethyl alcohol for 20 minutes each at ratios of 1:2, 2:1, and finally 100\% HDMS until fully evaporated (up to $1 \mathrm{hr}$ at RT). Scaffolds were mounted onto aluminium stubs (Ted Pella Inc., USA) and sputter coated with gold for 2 minutes (E5100 sputter coater, Polaron Equipment Inc, UK). Scaffolds were viewed in a Philips XL30 scanning electron microscope (Philips, Netherlands) with representative images taken to evaluate surface structures and morphology. 


\section{Statistical analysis}

Membrane groups were compared statistically using a one-way ANOVA with Tukey post hoc analysis (at the 0.05 significance level; GraphPad Prism 7; GraphPad Software, Inc., La Jolla, California, USA). Raw data for ANOVA comparison was assessed for normality using a Shapiro-Wilk normality test (0.05 significance level). Where data was not normally distributed, the whole data set was $\log _{10}$ transformed then retested to confirm normality before ANOVA on the transformed data. 


\section{Results}

\section{SF membrane casting}

The cross-linked film formation were obtained by a gelation phase of the SF solution in formic acid by photo-cross-linking and the following evaporation of formic acid and the resulting structural collapse of the gel. The photo-cross-linking method rapidly induced the formation of a viscous gel within seconds after exposure to a light source (Figure 1A, B). If a portion of the cast solution was selectively exposed to the light source, e.g. using a filter or a laser, only the irradiated area formed a gel, while the rest of the cast solution remained in a liquid state and could be easily washed away with water (Figure 1C, D). After cross-linking, the SF gels were dried in air to form a solid membrane. During the drying phase, the volume of the gels reduced, passing from a $3.5 \mathrm{ml} \mathrm{SF}$ solution casted in a $5 \mathrm{~cm}$ diameter culture dish to a thin solid membrane after drying overnight. Flat membranes photo-cross-linked in the base of plastic cell culture dishes were adherent, but could be cast on thermoplastic film (Parafilm) to allow easy release and handling. The formation of pure SF membranes (P-SM) by drying SF solution happened slowly at room temperature: the organization of the protein in solution into a solid network occurred naturally by drying and an intermediate phase of gel was not observed. After the drying process was complete, a solid, transparent SF membrane was collected (Figure 1E). All of the SF membranes produced had good transparency (Figure 1E, F). P-SM appeared as a solid and transparent thin film (Figure 1E), while the presence of ruthenium induced a strong yellow hue (Figure 1F). Both the pure SF (P-SM) and cross-linked SF (CL-SM) formed solid membranes that became brittle when left over time at room temperature. Although sampling was possible, the membranes easily cracked when stretched or bent. The addition of $40 \% \mathrm{w} / \mathrm{w}$ glycerol allowed the membrane to become plasticized, able to stretch or bend without cracking. After the drying process was completed, the thickness of the membranes in their dry state was measured: P-SM 105.9 $\pm 17.1 \mu \mathrm{m}$; P-SM-G $115.1 \pm 4.7 \mu \mathrm{m}$; CL-SM 106.3 $\pm 14.1 \mu \mathrm{m}$; CLSM-G $122.4 \pm 2.2 \mu \mathrm{m}$. 


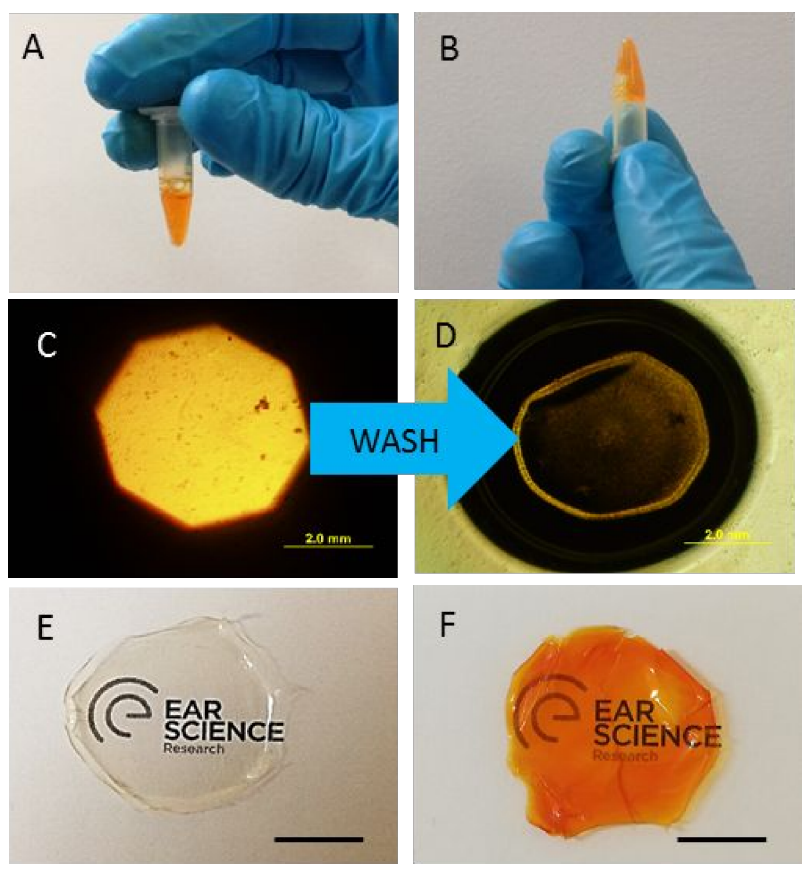

Figure 1: Cross-linking of SF to form membranes. Initially a SF solution was mixed with photoreactive agents to form a photo-sensitive SF liquid (A). When the solution was subsequently exposed to light, it turned into a viscous gel (B). Alternatively, a portion of the SF solution could be photo-cross-linked by exposing a selected area to the light source (i.e. here by partially closing the diaphragm of an optical microscope, with the light source turned on. C), while the unreacted portion could be washed away afterwards to leave the solid SF pattern (D). When dried, un-cross-linked membranes were colourless and transparent (E), while photo-crosslinked ones presented a vivid yellow hue of similar transparency (F). Scale bars: C, D: $2 \mathrm{~mm}$; E, F: $1 \mathrm{~cm}$;

\section{Silk fibroin secondary structure}

Secondary structure of membranes was evaluated by FTIR spectroscopy (Figure 3). In general, the spectra taken from the different membrane compositions did not show significant changes (Figure 3a), with the exception of two additional peaks present in just the CL-SM-G membranes: a peak at $1716.6 \mathrm{~cm}^{-1}$ and the peak at $1162 \mathrm{~cm}^{-1}$, which was more prominent in this sample compared with the other spectra (Figure 3b). The exact identity of the $1163 \mathrm{~cm}^{-1}$ peak is not clear, it could represent amine C-N stretching $\left(1250\right.$ to $\left.1020 \mathrm{~cm}^{-1}\right)$ or C-O stretching from an aliphatic ether (1150 to $\left.1085 \mathrm{~cm}^{-1}\right)$ or from a tertiary alcohol $\left(1205-1124 \mathrm{~cm}^{-1}\right)$. The second peak at $1716.6 \mathrm{~cm}^{-1}$ sits within the region associated with $\mathrm{C}=\mathrm{O}$ stretching, for example 
from carboxylic acid (1720-1706 cm-1; https://chem.libretexts.org). The reason for the appearance of these peaks is unclear and warrants further investigation. Since the changes are only associated with the combination of cross-linking with glycerol, it is possible that the ruthenium catalyst has covalently attached some glycerol molecules into the silk membrane and that the new $\mathrm{C}-\mathrm{O}$ and $\mathrm{C}=\mathrm{O}$ stretching is associated with incorporation of oxygen molecules from the hydroxyl groups of the glycerol.

When studying the secondary structure of fibroin by deconvoluting the amide I region (Figure $3 c$, Table 2) the P-SM and CL-SM had similar crystalline content ( $\beta$-sheets and turns) as well as a low content of amorphous structures (random coil and $\alpha$-helix). FTIR analysis showed that P-SM and CL-SM had similar spectra, with characteristic peaks in the two $\beta$-sheet regions (1616 to $1637 \mathrm{~cm}^{-1}$ and 1697 to $1703 \mathrm{~cm}^{-1}$ ), while they lacked a defined peak in the random coil/a-helix region.

The addition of glycerol in both formulations increased the SF $\beta$-sheet content (Table 2). The CL-SM-G contained the highest proportion of $\beta$-sheet area among all the membranes (almost $70 \%)$, significantly higher than P-SM-G $(\mathrm{p}<0.05)$, but had a significant drop in the $\beta$-turn content $(\mathrm{p}<0.001)$ from $12.1 \%$ to $5.1 \%$ in the CL-SM-G. Regarding the amorphous structure content, the addition of glycerol reduced the level of random coil and $\alpha$-helix (Table 2). 


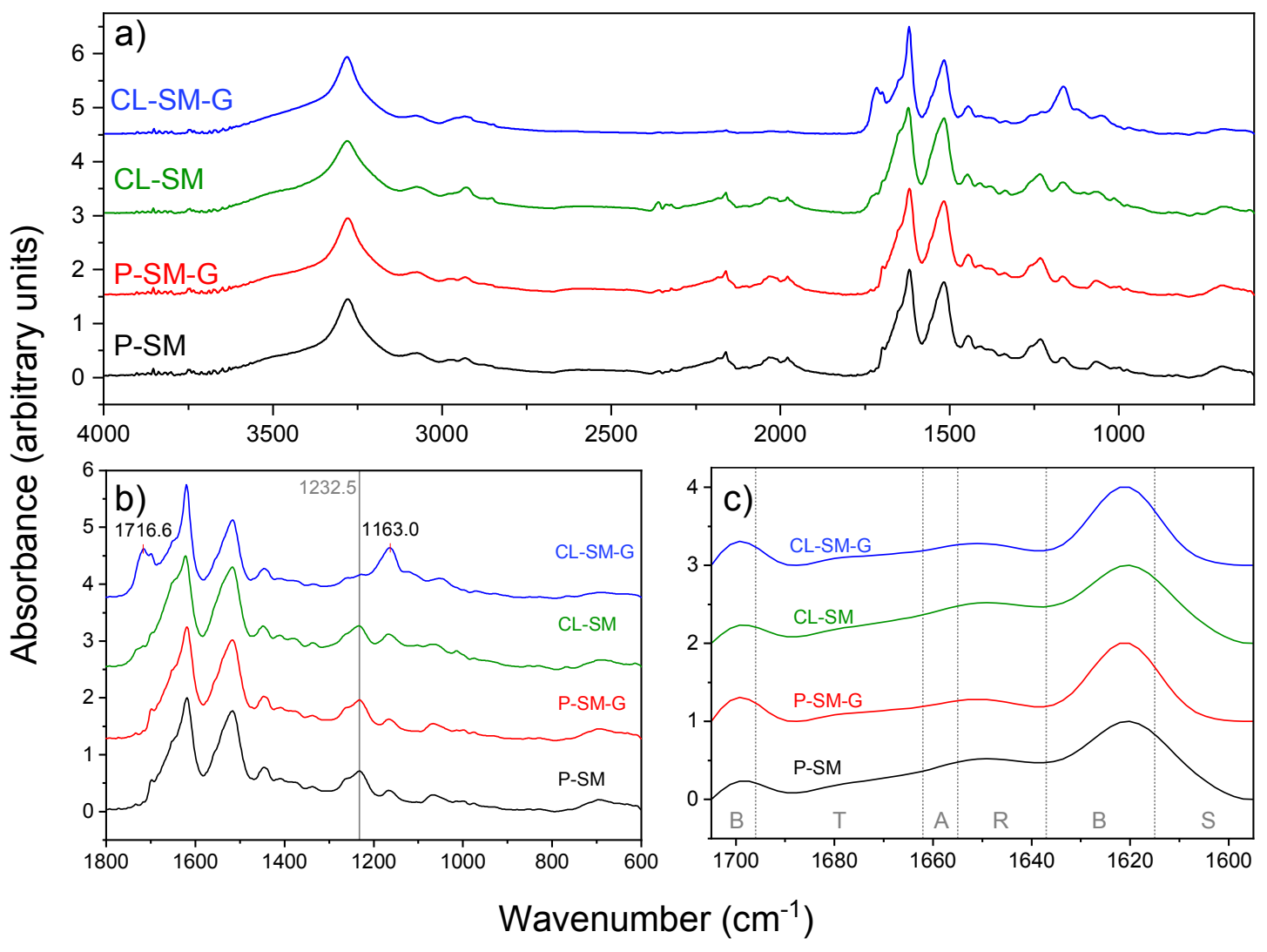

Figure 3: FTIR spectra of the 4 membrane compositions, including the entire scan range (a), frequencies below $1800 \mathrm{~cm}^{-1}$ and the deconvoluted spectra within the amide I region associated with protein secondary structure (c) Regions between dotted lines represent the different secondary structure motifs: $\mathrm{B}=\beta$-sheet, $\mathrm{T}=$ turn, $\mathrm{A}=\alpha$-helix, $\mathrm{R}=$ random coil, $\mathrm{S}=$ side chain. All plots represent the mean of 4 measurements per membrane (edge of the membrane, top surface, edge of the membrane bottom surface, centre of the membrane top surface, centre of the membrane bottom surface) over 3 separate films, for a total of 12 measurements per membrane type. 
Table 2: Percentage of secondary structure components for different SF membranes. A total of 3 membranes of each type were measured, with two measurements made per membrane (one in the centre and one near the edge of the membrane). Values represent mean \pm standard deviation of 12 total measurements

\begin{tabular}{lllll} 
Sample & P-SM & P-SM-G & CL-SM & CL-SM-G \\
\hline Side chain & $5.4 \pm 4.5$ & $2.0 \pm 2.3$ & $2.6 \pm 0.7$ & 0 \\
\hline$\beta$-Sheet & $50.8 \pm 8.1$ & $62.0 \pm 6.5$ & $45.0 \pm 2.7$ & $69.4 \pm 0.7$ \\
\hline Random Coil & $34.1 \pm 6.5$ & $26.7 \pm 7.6$ & $34.1 \pm 4.1$ & $26.1 \pm 1.0$ \\
\hline$\alpha$-Helix & 0 & 0 & $6.2 \pm 3.9$ & 0 \\
\hline Turn & $9.7 \pm 2.4$ & $9.3 \pm 2.8$ & $12.1 \pm 2.7$ & $5.1 \pm 1.3$ \\
\hline
\end{tabular}

\section{Mechanical properties of cross-linked films}

The addition of crosslinking made no impact to any of the mechanical properties of the membranes that had no glycerol (P-SM vs CL-SM; Table 3). The addition of glycerol resulted in a drop $(\mathrm{p}<0.0001)$ in the ultimate tensile strength and Young's modulus and an increase $(p<0.0001)$ in maximum elongation. This falls in line with previous results for SF membranes 27 and is expected due to the role of glycerol as a plasticiser. When comparing the membranes that contained glycerol (P-SM-G vs CL-SM-G), the addition of crosslinking led to a significantly higher tensile strength $(\mathrm{p}<0.0001)$ and maximum elongation $(\mathrm{P}<0.0001)$ but statistically similar Young's modulus ( $\mathrm{p}=0.962)$. In summary, although the addition of crosslinking to the pure SF membranes had no impact on mechanical properties, when cross-linking was used in combination with glycerol, the resulting membranes were significantly stronger and more ductile but without any unwanted increase in stiffness. 
Table 3: Tensile mechanical properties of cross-linked SF films compared with glycerol plasticised, cross-linked SF films. Values represent the mean \pm standard deviation of at least 20 strips across 3 membranes (sample size listed in the table). Superscript symbols indicate statistical grouping - values with the same letter are statistically similar $(\mathrm{P}>0.05)$.

\begin{tabular}{|c|c|c|c|c|}
\hline Sample & P-SM & P-SM-G & CL-SM & CL-SM-G \\
\hline \multicolumn{5}{|l|}{ Ultimate } \\
\hline Tensile & $58.9 \pm 17.8^{\mathrm{a}}$ & $12.9 \pm 5.4^{\mathrm{b}}$ & $63.4 \pm 9.7^{\mathrm{a}}$ & $20.4 \pm 6.8^{c}$ \\
\hline \multicolumn{5}{|l|}{ Strength ${ }^{21}$} \\
\hline $\begin{array}{l}\text { Young's } \\
\text { modulus (GPa) }\end{array}$ & $3.5 \pm 0.9^{\mathrm{a}}$ & $0.3 \pm 0.1^{\mathrm{b}}$ & $3.3 \pm 0.5^{\mathrm{a}}$ & $0.3 \pm 0.1^{\mathrm{b}}$ \\
\hline Elongation (\%) & $2.32 \pm 0.4^{\mathrm{a}}$ & $122.3 \pm 47.4^{\mathrm{b}}$ & $3.2 \pm 0.5^{\mathrm{a}}$ & $209.6 \pm 43.7^{\mathrm{c}}$ \\
\hline Sample size & 20 & 37 & 21 & 26 \\
\hline
\end{tabular}

In vitro enzymatic degradation assay

An accelerated protease degradation assay for SF membranes was developed using optical coherence tomography (OCT). Using serial volumetric imaging, the P-SM-G and CL-SM-G thickness pattern and their morphologic changes (Figure 4) were reported during enzymatic degradation up to 24 hours. Both types of membrane showed a homogeneous thickness in their dry state (Figure 4). When exposed to accelerated degrading conditions, the P-SM-G gradually degraded primarily from the cut edges, rather than in thickness. At 6 hours of incubation the remaining portion of the membrane had folded and by bi-dimensional scans a local increased thickness could be detected across a smaller area, and then a rapid degradation was complete by 12 hours. In contrast, the CL-SM-G showed a homogeneous thickness over time, with degradation limited to etching on the edge of the membrane observed at 24 hours of incubation. 

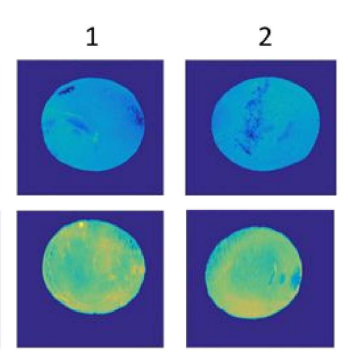
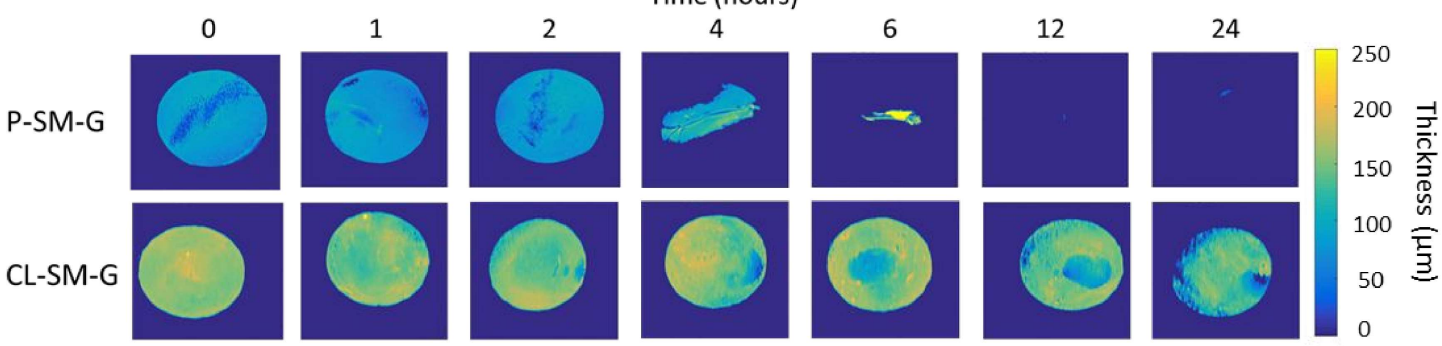

Figure 4: Optical coherence tomography analysis of SF membrane thickness during accelerated in vitro degradation. The images show the same pure SF membrane and photo-cross-linked SF membrane, both including glycerol, over time during the accelerated in vitro degradation assay. Samples reported here are representative of the two different sample groups utilized.

By integrating thickness over the surface area, the volume of each sample was calculated, and volume degradation was analysed (Figure 5). Protease-free control treatments, incubated in just PBS, did not show any significant volume changes during the 24 hours of incubation, except for the CL-SM-G that lost $20 \%$ of its initial volume in the first 4 hours, presumably due to glycerol leaching ${ }^{26}$. Results showed a significantly different volume degradation rate depending on the type of film. The P-SM exposed to protease solution degraded significantly faster than the other formulations ( $\mathrm{p}<0.001$ ): their volume was reduced by $90 \%$ within the first hour of incubation, and the membranes were reduced to debris that could not be imaged for volume analysis by the second hour of incubation. In comparison, the CL-SM cross-linked SF membranes degraded more slowly, lasting up to 6 hours of incubation $(\mathrm{p}<0,001)$. In the first two hours, the volume of CL-SM was reduced by $15 \%$, after that a rapid degradation was observed that led to complete dissolution of the samples within 6 hours of treatment. The PS$\mathrm{M}-\mathrm{G}$ incubated in protease solution rapidly degraded even after only two hours of incubation with the protease solution, the volume was reduced by $40 \%$ after 4 hours of incubation and by $94 \%$ after 6 hours, then debris was not visible after 12 hours. The degradation of CL-SM-G was significantly slower than the other formulations, as these membranes lost $25 \%$ of the initial volume in 12 hours, similar to protease free controls, and then kept constant to 24 hours incubation. 


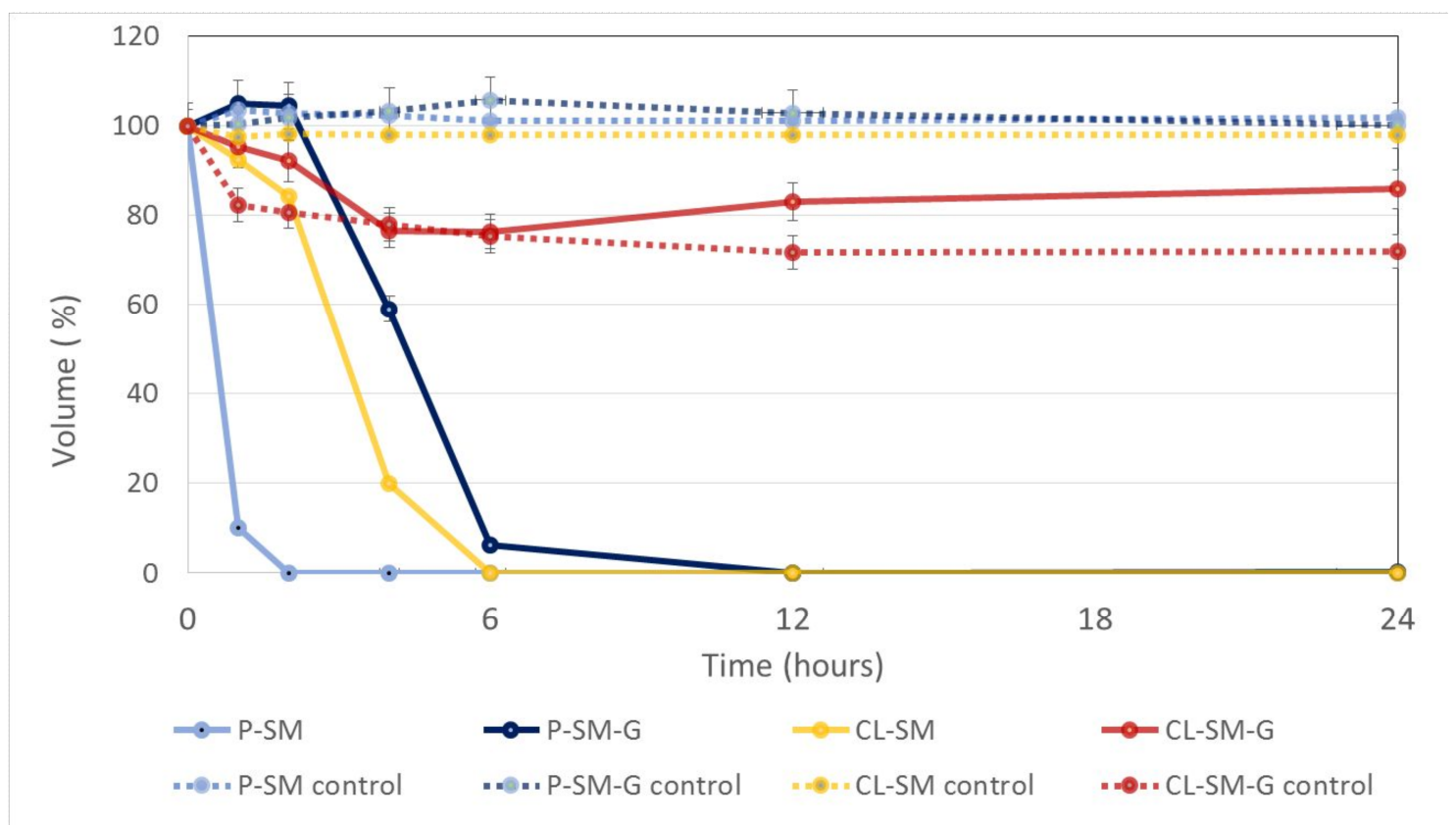

Figure 5: SF membrane volumetric degradation analysis by optical coherence tomography. The plot reports the volumetric variation of different SF membranes over time during accelerated in vitro enzymatic degradation assay (solid lines) and their enzyme-free controls (dashed lines). Samples were incubated in a solution of $1 \mathrm{mg} / \mathrm{ml}$ protease XIV from S. griseus in PBS for 24 hours at $37^{\circ} \mathrm{C}$. Controls were treated in the same conditions, but without protease present in the incubation solution.

\section{Cytocompatibility}

To assess their viability for tissue engineering applications, CL-SM-G were tested for in vitro cell culture using human tympanic membrane keratinocytes (hTMk), in order to explore use of cell monolayers on a surface ${ }^{49}$.

After 24 hours or 7 days of culturing the scaffold with the cells growing on it were fixed and stained with DAPI to observe the status of cell density and morphology on the fluorescence optical microscope (Figure 6 A, B). Generally, keratinocytes showed rapid adherence and after overnight incubation were found to be distributed on the surface of SF films in clusters, similar to the level on adjacent tissue culture plastic (data not shown). The cells successfully engrafted and proliferated on the CL-SM-G membranes: after 24 hours cells were found to be organized 
in small clusters (Figure 6A) over the culture well presenting their typical rounded shape. After one week of incubation they successfully formed a near-confluent monolayer of cells on the SF film (Figure 6B). Significant cell cycling (mitotic figures) was observed to be continuing at one week of cell culture (Figure $6 \mathrm{C}$, arrow heads).
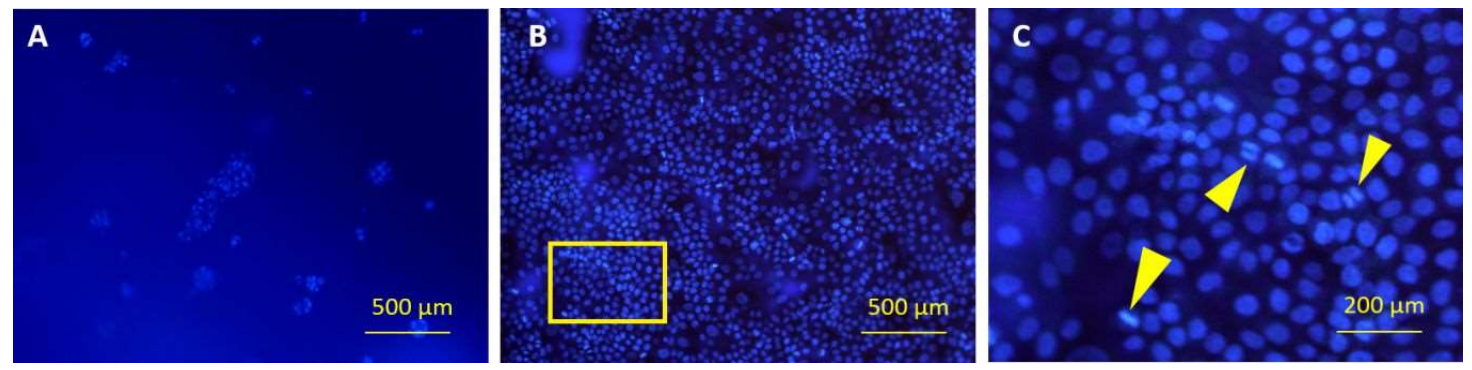

Figure 6: Fluorescence micrographs of human tympanic membrane keratinocytes engrafted and cultured on photo-cross-linked SF membrane. A, B: Cells were seeded and cultured on a photo-cross-linked SF membrane, and afterwards were stained with DAPI after 24 hours (A) or 7 days (B). Panels A and B indicate an active proliferation of the cells, growing over the SF membrane. Panel $\mathrm{C}$ shows an enlargement of the yellow box in panel $\mathrm{B}$, where numerous mitotic figures (arrow heads) could be observed after 7 days of cell culture (C). Scale bars are indicated. 


\section{Discussion}

Our findings suggest that it is possible to regulate the degradation rate of SF material through modifications to both crystalline and amorphous regions, using a combination of dityrosine photo-cross-linking and glycerol addition.

A previous work by Whittaker et al. ${ }^{5}$ described the use of ruthenium cross-linking of silk in water, primarily as a method to generate hydrogel materials. While this paper did describe the production of membranes by drying the cross-linked hydrogels, no attempt was made to assess the stability or degradation rate of these membranes or hydrogels. The results in this study demonstrate that membranes made with cross-linking without glycerol degraded within 2 hours in protease solution. This is in contrast with the novel composition proposed in this work (silk from formic acid, with glycerol + cross-linking), which lost less than $20 \%$ of its initial mass within 24 hours. These results build on our previous findings of the role of glycerol in combination with silk in formic acid as a method to create flexible yet slowly degrading membranes ${ }^{11}$. Our findings have demonstrated that only this combination, which has not been proposed previously, leads to the exceptional resistance to enzymatic attack - both cross-linked silk in formic acid (without glycerol) and formic acid silk with glycerol (without cross-linking) resulted in membranes that completely degraded within 6 to 12 hours. As represented in the proposed mechanism in Figure 7, the use of physical cross-linking (resulting from formic acid, enhanced by glycerol) and chemical cross-linking work synergistically to provide this enhanced resistance to degradation. We believe that this key advantage is vital for applications that require long term mechanical support.

The control of a biomaterial degradation profile is, in fact, a key aspect for its use in biomedical applications, impacting the quality and rate of tissue regeneration ${ }^{50}$. Generally, the crystalline structure of SF, formed by anti-parallel $\beta$-sheets, has a principal role in determining the SF strength and resistance to degradation by forming strong intra- and inter-molecular hydrogen bonds ${ }^{28}$. Several studies have reported the tuneable degradation of SF materials solely in relation to their level of $\beta$-sheets and the distribution and packing of its crystalline and amorphous domains $24,27,43-44$. In this work, the photo-cross-linked SF membranes showed an increased resistance to in vitro enzymatic degradation compared with non-cross-linked ones, but the analysis of the secondary structure did not show a significant difference in the $\beta$-sheet level between the two types of membranes. It appears, therefore, that the ruthenium-mediated 
photo-cross-linking results in an increased enzymatic degradation resistance of the SF without interfering with its self-assembly $\beta$-sheet motifs.

Photo-cross-linking SF using a ruthenium based-method induces the formation of covalent bonds between the its tyrosine residues, that constitute about $10 \%$ of the $\beta$-sheets- forming repeated units in the crystalline regions of $\mathrm{SF}^{19}$, but also the $5.3 \mathrm{~mol} \%$ in SF overall structure ${ }^{51}$. In fact, the amorphous region sequences is known to include tyrosine residues ${ }^{11,52}$, although its sequence and organization is not exhaustive ${ }^{53}$. As summarised in Figure 7, we hypothesise that tyrosine photo-cross-linking occurs in the amorphous region as well, affecting the mechanical properties and degradation rate of the SF membranes: when exposing the SF to a protease solution, the degradation affects preferentially the amorphous sites (Figure 7A), mainly because these sites are less dense, more solvated, prone to swelling and allow better access of the protease to its active site ${ }^{54}$. The ruthenium photo-cross-linking reaction could protect these sites from enzymatic degradation by holding adjacent protein chains together, reducing their mobility and preventing the protease access to the binding sites (Figure 7B). A similar hypothesis was recently proposed in a theoretical model using controlled light-induced radical grafting that cross-links the SF amorphous region, with the formation of covalent bonds among the amino acids of the SF, affecting the degradation rate in vitro of the biomaterial ${ }^{55}$. However, the use of the sole photo-cross-link on SF, results in a brittle dry material, which has limited applicability as a biomaterial. The use of a plasticizing agent, like glycerol, has been previously reported as a biocompatible method to improve the flexibility of the SF based biomaterials ${ }^{26-27}$, as well as to increase the level of $\beta$-sheets of the $\mathrm{SF}^{24}$. The glycerol, due to its hydroscopic nature, retains small portions of water within the drying material, reduces the attractive forces between adjacent protein chains, therefore, slowing the drying process and induces the self-assembly and spatial lodgement of higher portion of $\beta$-sheets ${ }^{27,}{ }^{56}$. The resulting glycerol-plasticized structure allows the protein chains to move past each other, inducing a drop in the membranes Young's modulus and an increase in the maximum elongation. However, in native SF fibers the elasticity of the material is usually caused by stretching of the amorphous domains (Figure 7C) ${ }^{57}$. The photo-cross-linking of SF in presence of glycerol increases significantly the elongation rate of the SF membrane, providing strong chemical cross-linkages in the SF amorphous region, which increase the amount of strain the material can experience before failure in tensile testing. The mechanical properties of the SF membranes were determined in a dry state, as the primary intended application for this materials is in the middle ear. While we believe this environment is humid, it is not fully 
hydrated, and the membranes will be exposed to air within the middle ear cavity on one side. In addition, testing the membranes while wet would leach out the glycerol ${ }^{26}$, changing the mechanical properties in a way that will not reflect the in vivo environment.

To test if the SF photo-cross-linked membranes were capable to support the target tissue regeneration, their cytocompatibility was evaluated in vitro. Human tympanic membranes keratinocytes were chosen to study the biocompatibility of the SF biomaterials, as these thin membranes could be suitable for long term replacement of the human tympanic membrane. Previous works reported separately that the use of formic acid ${ }^{24}$, glycerol ${ }^{58}$ and ruthenium/SPS cross-link ${ }^{5}$ does not impair the SF materials biocompatibility. The combination of these treatments in the current formulation allowed the cell engraftment and proliferation over time (Figure 6) on the CL-SM-G in their wet state.

As a further scope, improving the manufacturing method to induce the photo-cross-link reaction could grant a higher degree of control of the SF structure together with the precise patterning of the percentage of SF that is cross-linked. While the SF solution is stable for long term at room temperature and under normal light exposure, a short-time exposure using a $22 \mathrm{~W}$ white lamp was used to induce the gelation of SF solution + photo-reagents. It is worth noticing that the light power required to induce the photo-cross-linking was significantly less than what reported previously ${ }^{5}$. Although the relationship between the light exposure and the rate of photo-cross-linking was not investigated in this work, the reaction can be induced by a low amount of energy, in a short time range and with a non-cytotoxic concentration of reagents. In fact, since there is no way of quenching the reaction without washing out the ruthenium, our understanding is that cross-linking will continue until all possible reaction sites have been saturated. This scenario opens hypothesis for further investigations on the regulation of this process though different sources of light exposure, with outcomes in selective patterning and additive manufacturing. Examples in literature have been reported using short-UV laser pulse ${ }^{59}$, or two-photon IR laser ${ }^{37}$, however those methods haven't been translated into a biomedical application yet.

As the field of biomedical applications moves into the territory of precision medicine, biomaterials that can be customized for the single patient have to be developed, in terms of morphology, physico-chemical characteristics and biocompatibility. Our SF based scaffold biomaterial with all these characteristics may likely fit tissue engineering and other biomedical 
applications that require longer term mechanical support, such as tympanic membrane repair or bone regeneration, providing robust and flexible support.

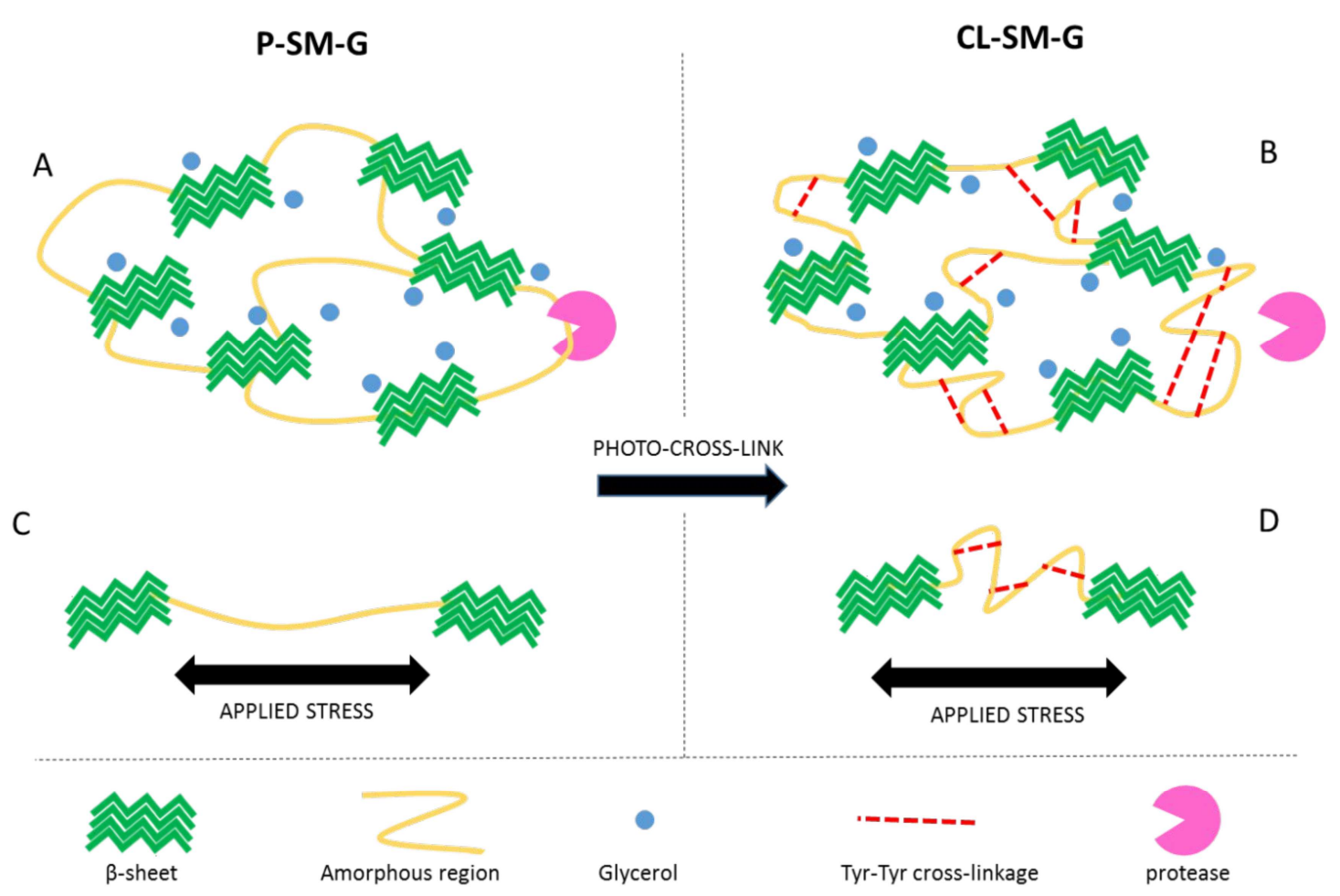

Figure 7: Graphical representation of the effect of photo-cross-linking in amorphous SF regions. When glycerol is included in SF composition, is dispersed among the protein chains, increasing their mobility and the content of $\beta$-sheet. During degradation assay, the protease tends to cleave the SF amorphous regions (A), as these are more swollen and solvated. When Tyr-Tyr photocross-link is induced in the SF amorphous regions (B), it tights the protein chains, making it more difficult for the protease to access the binding sites in those regions. Moreover, when a stress is applied to deform the SF membrane (C)

, the elongation is related to the stretching of the amorphous regions. The Tyr-Tyr bonds in the amorphous region formed by the photo-cross-linking limit the stretching of the amorphous regions (D) resulting in a diminished elongation rate of the SF membrane. 


\section{Conclusion}

Using a combination of ruthenium photo-initiator, formic acid as a solvent and the addition of glycerol, it was possible to generate SF membranes with enhanced $\beta$-sheet content as well as the introduction of chemical cross-links. This combination delivered exceptional resistance to enzymatic degradation, with minimal effect on membrane mechanical properties and without affecting the biocompatibility of the material in vitro. This represents a feasible approach to produce SF-based biomaterials designed for tissue engineering applications.

\section{Funding:}

This research was supported by the Australian Research Council Research Hub for Future Fibers (IH140100018) and funded by the Australian Government.

\section{Supporting information:}

Analysis of the ruthenium leaching from the C-SM-G over time (Supplementary Figure 1). 
For Table of Contents Use Only

Enhancing resistance of silk fibroin material to enzymatic degradation by cross-linking both crystalline and amorphous domains

Filippo Valente ${ }^{\mathrm{a} *}$, Benjamin J. Allardyce ${ }^{\mathrm{b} *}$, Matt S. Hepburn ${ }^{\mathrm{c}, \mathrm{d}}$, Philip Wijesinghe ${ }^{\mathrm{c}, \mathrm{d}, \mathrm{e}}$, Sharon

L. Redmond ${ }^{\mathrm{a}}$, Jingyu Chen ${ }^{\mathrm{b}}$, Brendan F. Kennedyc,d, Rangam Rajkhowa ${ }^{\mathrm{b}}$, Marcus D. Atlas $^{\mathrm{a}}$, Xungai Wang ${ }^{\mathrm{b}}$ and Rodney J. Dilley, , ,**

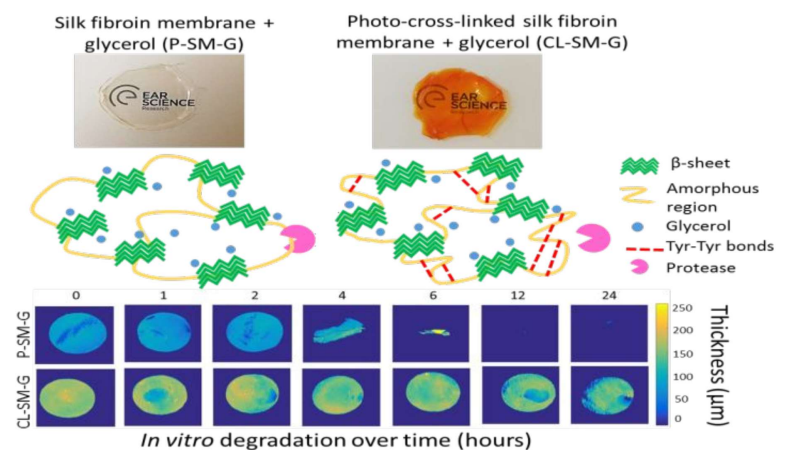




\section{Bibliography}

1. Qi, Y.; Wang, H.; Wei, K.; Yang, Y.; Zheng, R. Y.; Kim, I. S.; Zhang, K. Q., A Review of Structure Construction of Silk Fibroin Biomaterials from Single Structures to Multi-Level Structures. Int J Mol Sci 2017, 18 (3). DOI: 10.3390/ijms18030237.

2. Kundu, B.; Rajkhowa, R.; Kundu, S. C.; Wang, X., Silk fibroin biomaterials for tissue regenerations. Adv Drug Deliv Rev 2013, 65 (4), 457-70. DOI: 10.1016/j.addr.2012.09.043.

3. Altman, G. H.; Diaz, F.; Jakuba, C.; Calabro, T.; Horan, R. L.; Chen, J.; Lu, H.; Richmond, J.; Kaplan, D. L., Silk-based biomaterials. Biomaterials 2003, 24 (3), 401-16.

4. Melke, J.; Midha, S.; Ghosh, S.; Ito, K.; Hofmann, S., Silk fibroin as biomaterial for bone tissue engineering. Acta Biomater 2016, 31, 1-16. DOI: 10.1016/j.actbio.2015.09.005.

5. Whittaker, J. L.; Choudhury, N. R.; Dutta, N. K.; Zannettino, A., Facile and rapid ruthenium mediated photo-crosslinking of Bombyx mori silk fibroin. Journal of Materials Chemistry B 2014, 2 (37), 6259-6270. DOI: 10.1039/C4TB00698D.

6. Allardyce, B. J.; Rajkhowa, R.; Dilley, R. J.; Xie, Z.; Campbell, L.; Keating, A.; Atlas, M. D.; von Unge, M.; Wang, X., Comparative acoustic performance and mechanical properties of silk membranes for the repair of chronic tympanic membrane perforations. J Mech Behav Biomed Mater 2016, 64, 6574. DOI: 10.1016/j.jmbbm.2016.07.017.

7. Tsai, I. L.; Hsu, C. C.; Hung, K. H.; Chang, C. W.; Cheng, Y. H., Applications of biomaterials in corneal wound healing. J Chin Med Assoc 2015, 78 (4), 212-7. DOI: 10.1016/j.jcma.2014.09.011.

8. Park, Y. R.; Ju, H. W.; Lee, J. M.; Kim, D. K.; Lee, O. J.; Moon, B. M.; Park, H. J.; Jeong, J. Y.; Yeon, Y. K.; Park, C. H., Three-dimensional electrospun silk-fibroin nanofiber for skin tissue engineering. Int J Biol Macromol 2016, 93 (Pt B), 1567-1574. DOI: 10.1016/j.ijbiomac.2016.07.047.

9. Turkkan, S.; Atila, D.; Akdag, A.; Tezcaner, A., Fabrication of functionalized citrus pectin/silk fibroin scaffolds for skin tissue engineering. J Biomed Mater Res B Appl Biomater 2018. DOI: 10.1002/jbm.b.34079.

10. Jose, R. R.; Raja, W. K.; Ibrahim, A. M.; Koolen, P. G.; Kim, K.; Abdurrob, A.; Kluge, J. A.; Lin, S. J.; Beamer, G.; Kaplan, D. L., Rapid prototyped sutureless anastomosis device from self-curing silk bioink. J Biomed Mater Res B Appl Biomater 2015, 103 (7), 1333-43. DOI: 10.1002/jbm.b.33312.

11. Lefevre, T.; Rousseau, M. E.; Pezolet, M., Protein secondary structure and orientation in silk as revealed by Raman spectromicroscopy. Biophys J 2007, 92 (8), 2885-95. DOI: 10.1529/biophysj.106.100339.

12. Keten, S.; Xu, Z.; Ihle, B.; Buehler, M. J., Nanoconfinement controls stiffness, strength and mechanical toughness of $\beta$-sheet crystals in silk. Nature Materials 2010, 9 (4), 359-367. DOI: http://www.nature.com/nmat/journal/v9/n4/abs/nmat2704.html\#supplementary-information. 
13. Minoura, N.; Tsukada, M.; Nagura, M., Physicochemical properties of silk fibroin membrane as a biomaterial. Biomaterials 1990, 11 (6), 430-434.

14. Jin, H. J.; Park, J.; Karageorgiou, V.; Kim, U. J.; Valluzzi, R.; Cebe, P.; Kaplan, D. L., Water-stable silk films with reduced $\beta$-sheet content. Advanced Functional Materials 2005, 15 (8), 1241-1247. DOI: 10.1002/adfm.200400405.

15. Allardyce, B. J.; Rajkhowa, R.; Dilley, R. J.; Redmond, S. L.; Atlas, M. D.; Wang, X., Glycerolplasticised silk membranes made using formic acid are ductile, transparent and degradation-resistant. Materials Science \& Engineering C-Materials for Biological Applications 2017, 80, 165-173. DOI: 10.1016/j.msec.2017.05.112.

16. Magoshi, J.; Magoshi, Y.; Nakamura, S., Physical properties and structure of silk. VII. Crystallization of amorphous silk fibroin induced by immersion in methanol. Journal of Polymer Science: Polymer Physics Edition 1981, 19 (1), 185-186. DOI: 10.1002/pol.1981.180190117.

17. Hu, X.; Shmelev, K.; Sun, L.; Gil, E. S.; Park, S. H.; Cebe, P.; Kaplan, D. L., Regulation of silk material structure by temperature-controlled water vapor annealing. Biomacromolecules 2011, 12 (5), 1686-1696. DOI: Doi 10.1021/Bm200062a.

18. Demura, M.; Asakura, T., Immobilization of glucose oxidase with Bombyx mori silk fibroin by only stretching treatment and its application to glucose sensor. 1989, 33 (5), 598-603. DOI: 10.1002/bit.260330513.

19. Zhou, C. Z.; Confalonieri, F.; Jacquet, M.; Perasso, R.; Li, Z. G.; Janin, J., Silk fibroin: structural implications of a remarkable amino acid sequence. Proteins 2001, 44 (2), 119-22.

20. Zhou, Q.; Cui, L.; Ren, L.; Wang, P.; Deng, C.; Wang, Q.; Fan, X., Preparation of a multifunctional fibroin-based biomaterial via laccase-assisted grafting of chitooligosaccharide. Int J Biol Macromol 2018, 113, 1062-1072. DOI: 10.1016/j.ijbiomac.2018.03.042.

21. Freddi, G.; Anghileri, A.; Sampaio, S.; Buchert, J.; Monti, P.; Taddei, P., Tyrosinase-catalyzed modification of Bombyx mori silk fibroin: grafting of chitosan under heterogeneous reaction conditions. J Biotechnol 2006, 125 (2), 281-94. DOI: 10.1016/j.jbiotec.2006.03.003.

22. Ribeiro, V. P.; Silva-Correia, J.; Goncalves, C.; Pina, S.; Radhouani, H.; Montonen, T.; Hyttinen, J.; Roy, A.; Oliveira, A. L.; Reis, R. L.; Oliveira, J. M., Rapidly responsive silk fibroin hydrogels as an artificial matrix for the programmed tumor cells death. PLoS One 2018, 13 (4), e0194441. DOI: 10.1371/journal.pone.0194441.

23. Partlow, B. P.; Hanna, C. W.; Rnjak-Kovacina, J.; Moreau, J. E.; Applegate, M. B.; Burke, K. A.; Marelli, B.; Mitropoulos, A. N.; Omenetto, F. G.; Kaplan, D. L., Highly tunable elastomeric silk biomaterials. Adv Funct Mater 2014, 24 (29), 4615-4624. DOI: 10.1002/adfm.201400526. 
24. Allardyce, B. J.; Rajkhowa, R.; Dilley, R. J.; Redmond, S. L.; Atlas, M. D.; Wang, X., Glycerolplasticised silk membranes made using formic acid are ductile, transparent and degradation-resistant. Mater Sci Eng C Mater Biol Appl 2017, 80, 165-173. DOI: 10.1016/j.msec.2017.05.112.

25. Pei, Y.; Liu, X.; Liu, S.; Lu, Q.; Liu, J.; Kaplan, D. L.; Zhu, H., A mild process to design silk scaffolds with reduced beta-sheet structure and various topographies at the nanometer scale. Acta Biomater 2015, 13, 168-76. DOI: 10.1016/j.actbio.2014.11.016.

26. Brown, J. E.; Davidowski, S. K.; Xu, D.; Cebe, P.; Onofrei, D.; Holland, G. P.; Kaplan, D. L., Thermal and Structural Properties of Silk Biomaterials Plasticized by Glycerol. Biomacromolecules 2016, 17 (12), 3911-3921. DOI: 10.1021/acs.biomac.6b01260.

27. Lu, S.; Wang, X.; Lu, Q.; Zhang, X.; Kluge, J. A.; Uppal, N.; Omenetto, F.; Kaplan, D. L., Insoluble and flexible silk films containing glycerol. Biomacromolecules 2010, 11 (1), 143-50. DOI: 10.1021/bm900993n.

28. Xiao, S.; Stacklies, W.; Cetinkaya, M.; Markert, B.; Grater, F., Mechanical response of silk crystalline units from force-distribution analysis. Biophys J 2009, 96 (10), 3997-4005. DOI: 10.1016/j.bpj.2009.02.052.

29. Song, P.; Xu, Z.; Lu, Y.; Guo, Q., Bio-Inspired Hydrogen-Bond Cross-Link Strategy toward Strong and Tough Polymeric Materials. Macromolecules 2015, 48 (12), 3957-3964. DOI: 10.1021/acs.macromol.5b00673.

30. Fancy, D. A.; Kodadek, T., Chemistry for the analysis of protein-protein interactions: Rapid and efficient cross-linking triggered by long wavelength light. Proceedings of the National Academy of Sciences 1999, 96 (11), 6020-6024. DOI: 10.1073/pnas.96.11.6020.

31. Elvin, C. M.; Carr, A. G.; Huson, M. G.; Maxwell, J. M.; Pearson, R. D.; Vuocolo, T.; Liyou, N. E.; Wong, D. C. C.; Merritt, D. J.; Dixon, N. E., Synthesis and properties of crosslinked recombinant proresilin. Nature 2005, 437 (7061), 999-1002. DOI: 10.1038/nature04085.

32. Elvin, C. M.; Brownlee, A. G.; Huson, M. G.; Tebb, T. A.; Kim, M.; Lyons, R. E.; Vuocolo, T.; Liyou, N. E.; Hughes, T. C.; Ramshaw, J.; Werkmeister, J. A., The development of photochemically crosslinked native fibrinogen as a rapidly formed and mechanically strong surgical tissue sealant. Biomaterials 2009, 30 (11). DOI: 10.1016/j.biomaterials.2008.12.059.

33. Vashi, A. V.; Werkmeister, J. A.; Vuocolo, T.; Elvin, C. M.; Ramshaw, J. A. M., Stabilization of collagen tissues by photocrosslinking. Journal of Biomedical Materials Research Part A 2012, 100A (9), 2239-2243. DOI: 10.1002/jbm.a.34164.

34. Murphy, A. R.; Kaplan, D. L., Biomedical applications of chemically-modified silk fibroin. J Mater Chem 2009, 19 (36), 6443-6450. DOI: 10.1039/b905802h. 
35. Zhou, C. Z.; Confalonieri, F.; Medina, N.; Zivanovic, Y.; Esnault, C.; Yang, T.; Jacquet, M.; Janin, J.; Duguet, M.; Perasso, R.; Li, Z. G., Fine organization of Bombyx mori fibroin heavy chain gene. Nucleic Acids Res. 2000, 28 (12), 2413-2419. DOI: 10.1093/nar/28.12.2413.

36. Knowlton, S.; Yenilmez, B.; Anand, S.; Tasoglu, S., Photocrosslinking-Based Bioprinting: Examining Crosslinking Schemes. 2017; Vol. 5. DOI: 10.1016/j.bprint.2017.03.001.

37. Dickerson, M. B.; Dennis, P. B.; Tondiglia, V. P.; Nadeau, L. J.; Singh, K. M.; Drummy, L. F.; Partlow, B. P.; Brown, D. P.; Omenetto, F. G.; Kaplan, D. L.; Naik, R. R., 3D Printing of Regenerated Silk Fibroin and Antibody-Containing Microstructures via Multiphoton Lithography. ACS Biomaterials Science \& Engineering 2017, 3 (9), 2064-2075. DOI: 10.1021/acsbiomaterials.7b00338.

38. Mustafa, F. H.; Jaafar, M. S., Comparison of wavelength-dependent penetration depths of lasers in different types of skin in photodynamic therapy. Indian Journal of Physics 2013, 87 (3), 203209. DOI: 10.1007/s12648-012-0213-0.

39. Lim, K. S.; Schon, B. S.; Mekhileri, N. V.; Brown, G. C. J.; Chia, C. M.; Prabakar, S.; Hooper, G. J.; Woodfield, T. B. F., New Visible-Light Photoinitiating System for Improved Print Fidelity in GelatinBased Bioinks. ACS Biomaterials Science \& Engineering 2016, 2 (10), 1752-1762. DOI: 10.1021/acsbiomaterials.6b00149.

40. Wang, Z.; Jin, X.; Dai, R.; Holzman, J. F.; Kim, K., An ultrafast hydrogel photocrosslinking method for direct laser bioprinting. RSC Advances 2016, 6 (25), 21099-21104. DOI: 10.1039/C5RA24910D.

41. Elvin, C. M.; Brownlee, A. G.; Huson, M. G.; Tebb, T. A.; Kim, M.; Lyons, R. E.; Vuocolo, T.; Liyou, N. E.; Hughes, T. C.; Ramshaw, J. A.; Werkmeister, J. A., The development of photochemically crosslinked native fibrinogen as a rapidly formed and mechanically strong surgical tissue sealant. Biomaterials 2009, 30 (11), 2059-65. DOI: 10.1016/j.biomaterials.2008.12.059.

42. Elvin, C. M.; Vuocolo, T.; Brownlee, A. G.; Sando, L.; Huson, M. G.; Liyou, N. E.; Stockwell, P. R.; Lyons, R. E.; Kim, M.; Edwards, G. A.; Johnson, G.; McFarland, G. A.; Ramshaw, J. A.; Werkmeister, J. A., A highly elastic tissue sealant based on photopolymerised gelatin. Biomaterials 2010, 31 (32), 8323-31. DOI: 10.1016/j.biomaterials.2010.07.032.

43. Zhang, L.; Liu, X.; Li, G.; Wang, P.; Yang, Y., Tailoring degradation rates of silk fibroin scaffolds for tissue engineering. J Biomed Mater Res A 2018. DOI: 10.1002/jbm.a.36537.

44. Numata, K.; Cebe, P.; Kaplan, D. L., Mechanism of enzymatic degradation of beta-sheet crystals. Biomaterials 2010, 31 (10), 2926-33. DOI: 10.1016/j.biomaterials.2009.12.026.

45. Hu, X.; Kaplan, D.; Cebe, P., Determining beta-sheet crystallinity in fibrous proteins by thermal analysis and infrared spectroscopy. Macromolecules 2006, 39 (18), 6161-6170. DOI: 10.1021/ma0610109. 
46. Rajkhowa, R.; Hu, X.; Tsuzuki, T.; Kaplan, D. L.; Wang, X., Structure and biodegradation mechanism of milled Bombyx mori silk particles. Biomacromolecules 2012, 13 (8), 2503-12. DOI: $10.1021 / \mathrm{bm} 300736 \mathrm{~m}$.

47. Redmond, S. L.; Levin, B.; Heel, K. A.; Atlas, M. D.; Marano, R. J., Phenotypic and genotypic profile of human tympanic membrane derived cultured cells. J Mol Histol 2011, 42 (1), 15-25. DOI: 10.1007/s10735-010-9303-5.

48. Khoonta, P.; Linsuwanon, P.; Posuwan, N.; Vongpunsawad, S.; Payungporn, S.; Poovorawan, Y., Prevalence and molecular characterization of human rhinovirus in stool samples of individuals with and without acute gastroenteritis. J Med Virol 2017, 89 (5), 801-808. DOI: 10.1002/jmv.24698.

49. Bi, H.; Jin, Y., Current progress of skin tissue engineering: Seed cells, bioscaffolds, and construction strategies. Burns Trauma 2013, 1 (2), 63-72. DOI: 10.4103/2321-3868.118928.

50. Lee, O. J.; Lee, J. M.; Kim, J. H.; Kim, J.; Kweon, H.; Jo, Y. Y.; Park, C. H., Biodegradation behavior of silk fibroin membranes in repairing tympanic membrane perforations. J Biomed Mater Res A 2012, 100 (8), 2018-26. DOI: 10.1002/jbm.a.33308.

51. Truong, M. Y.; Dutta, N. K.; Choudhury, N. R.; Kim, M.; Elvin, C. M.; Nairn, K. M.; Hill, A. J., The effect of hydration on molecular chain mobility and the viscoelastic behavior of resilin-mimetic protein-based hydrogels. Biomaterials 2011, 32 (33), 8462-8473. DOI: https://doi.org/10.1016/j.biomaterials.2011.07.064.

52. Elliott, W. H.; Bonani, W.; Maniglio, D.; Motta, A.; Tan, W.; Migliaresi, C., Silk Hydrogels of Tunable Structure and Viscoelastic Properties Using Different Chronological Orders of Genipin and Physical Cross-Linking. ACS Appl Mater Interfaces 2015, 7 (22), 12099-108. DOI: 10.1021/acsami.5b02308.

53. Asakura, T.; Yao, J.; Yamane, T.; Umemura, K.; Ulrich, A. S., Heterogeneous structure of silk fibers from Bombyx mori resolved by 13 C solid-state NMR spectroscopy. J Am Chem Soc 2002, 124 (30), 8794-5.

54. Arai, T.; Freddi, G.; Innocenti, R.; Tsukada, M., Biodegradation of Bombyx mori silk fibroin fibers and films. Journal of Applied Polymer Science 2003, 91 (4), 2383-2390. DOI: 10.1002/app.13393. 55. Maziz, A.; Leprette, O.; Boyer, L.; Blatché, C.; Bergaud, C., Tuning the properties of silk fibroin biomaterial via chemical cross-linking. Biomedical Physics \& Engineering Express 2018, 4 (6), 065012. DOI: 10.1088/2057-1976/aae3b2.

56. Coupland, J. N.; Shaw, N. B.; Monahan, F. J.; Dolores O'Riordan, E.; O'Sullivan, M., Modeling the effect of glycerol on the moisture sorption behavior of whey protein edible films. Journal of Food Engineering 2000, 43 (1), 25-30. DOI: 10.1016/S0260-8774(99)00129-6. 
57. Su, I.; Buehler, M. J., Nanomechanics of silk: the fundamentals of a strong, tough and versatile material. Nanotechnology 2016, 27 (30). DOI: Artn 302001

10.1088/0957-4484/27/30/302001.

58. Vepari, C.; Kaplan, D. L., Silk as a biomaterial. Progress in Polymer Science 2007, 32 (8-9), 9911007. DOI: 10.1016/j.progpolymsci.2007.05.013.

59. Sun, Y. L.; Li, Q.; Sun, S. M.; Huang, J. C.; Zheng, B. Y.; Chen, Q. D.; Shao, Z. Z.; Sun, H. B., Aqueous multiphoton lithography with multifunctional silk-centred bio-resists. Nat Commun 2015, 6, 8612. DOI: $10.1038 /$ ncomms9612. 